\title{
$\alpha-\omega$ effect and recurrent changes of galactic cosmic rays intensity
}

\author{
Agnieszka Gil ${ }^{1}$ \\ Institute of Mathematics and Physics, Siedlce University, 3 Maja 54, 08-110 Siedlce, Poland \\ E-mail: gilaluph.edu.pl \\ Michael V. Alania \\ Institute of Mathematics and Physics, Siedlce University, 3 Maja 54, 08-110 Siedlce, Poland \\ and Institute of Geophysics, Tbilisi State University, Tbilisi, Georgia \\ E-mail: alanialuph.edu.pl
}

We recognize a quasi-recurrence with duration of 3 to 4 Carrington rotations period (3-4 CRP) in changes of amplitudes of the 27-day variation of galactic cosmic rays (GCR) intensity, solar activity (SA) and solar wind (SW) parameters, as well. We attribute this phenomenon to the presence of a spatial topological structure (STS) of the solar magnetic field. STS is created by $\alpha-\omega$ effect in the inner solar atmosphere, from photosphere to lower corona. STS exists due to the asymmetry of solar dynamo and solar differential rotation. Studying this phenomenon by the aid of spectral analysis methods we found an existence of the quasi-periodicities with the duration shorter than, and longer than the 3-4 CRP. This 3-4 CRP quasi-periodicity mode corresponds to the extreme interval $\Delta \mathrm{t}$ of differential rotation periods, $\Delta \mathrm{t}=$ 35 days -25 days (from poles to equator of the Sun, respectively). We assume also that the process like to $\alpha-\omega$ appears in time intervals corresponding to the transitional differential rotation periods, as well, e.g. $\Delta \mathrm{t}_{1}=26$ days -25 days, $\Delta \mathrm{t}_{2}=27$ days -25 days, and so forth, to $\Delta \mathrm{t}=35$ days -25 days. We assume that extensive range of quasi- periodicities of GCR intensity, SW and SA parameters can be related to the combined influence of turbulent solar dynamo and solar differential rotation. Thus, STS should be very complex pattern containing a broad modes of oscillations responsible for various types of quasiperiodicities. So, we believe that it is possible to find an evidence that any alternation: periodical or nonperiodical in GCR intensity and in parameters of solar activity and solar wind is strongly related with temporal changes of the various scales of STS.

35th International Cosmic Ray Conference - ICRC2017

10-20 July, 2017

Bexco, Busan, Korea

\section{${ }^{1}$ Speaker}




\section{Introduction}

The solar dynamo acts in the large scale periodicities; among them, the most obvious, morphologically linked with solar dynamo are 11-year and 22-year cycles (e.g. [1]). In [2] based on cosmogenic radionuclides analysis are considered long-lasting periodicities, amongst them 87-years and its multiplicities; there also is found an existence of mid-term quasi-recurrences. The quasi-biennial oscillations appearing stronger near the solar maximum are broadly discussed in connection with solar dynamo (e.g. [3-4]). In [5] using helioseismological data was found very clear periodicity $\sim 1.3$-years in the solar rotation tempo around the tachocline in the convective zone, which vanished after the year 2000 and didn't return, yet [6]. In [7] was stated that poloidal magnetic field has both: regular recurrences in the dynamo cycles and irregular changes in shorter time scales, analogous to the solar rotation period. An interesting review of other quasi-periodicities appearing in the Sun's behavior can be found in (e.g. [8-9]). Studying long period changes in [10] has been presented some unspecified torque effect on the assumed non-axisymmetric tachocline being a new input in the subject of possible planetary impact on the solar dynamo. In [11-13] was found a new class of quasi-periodicities, i.e. a distinct recurrence remaining three to four Carrington rotations period (3-4 CRP) in the amplitudes of the 27-day variations of the galactic cosmic ray (GCR) intensity, parameters of solar activity (SA) and solar wind (SW). The noticed 3-4 CRP quasi-recurrence is connected with the periodic configuration of the solar magnetic field. It exposes owing to the conversion of the poloidal magnetic field into the toroidal, due to the solar magnetic dynamo and differential rotation of the Sun $(\alpha-\omega$ effect).

We suggest an existence of a spatial topological magnetic structure with life-time of about 3 to 4 solar rotations (with lower and higher harmonics of the 3-4 CRP) on the Sun's photosphere (most apparent a current sheet type structure) created by the joint effect of turbulent solar dynamo and differential rotation of the Sun. A source of creation of that spatial topological magnetic structure is the $\alpha-\omega$ effect. We consider a topological structure as an area of the toroidal magnetic field lines originating in the helioequatorial region and being fully wrapped around the Sun, up to reaching the heliolongitudes with the origin of the poloidal magnetic field, from which the toroidal field was generated.

The suggested is dealt to the extreme interval $\Delta t$ of differential rotation periods, $\Delta t=25-35$ days (Sun's rotation period in equatorial region equals, $\mathrm{t}=25$ days, while $\mathrm{t}=35$ days in poles region). We assume that the $\alpha-\omega$ process appears in time intervals corresponding to the intermediate differential rotation periods, as well, e.g. $\Delta \mathrm{t}_{1}=25-26, \Delta \mathrm{t}_{2}=25-27$, and so on, up to $\Delta \mathrm{t}=25-35$ days including practically unlimited quasi-periodicities caused by a superposition of the basic modes, corresponding to various quasi-periodicities. Going to forth, one can suggest that different combination of basic modes of $\Delta t$ and their superposition can be considered as an origin of a great of majority types of quasi-periodical changes of GCR intensity, parameters of solar wind and solar activity.

Unfortunately, we exactly do not know yet what parameter or group of parameters characterizing the flexible topological structure created by the $\alpha-\omega$ effect is responsible for the 3-4 CRP cycling (including its lower and higher harmonics) of the amplitudes of the 27-day variations of the galactic cosmic ray intensity and various parameters of solar activity and solar wind. As a first approach there can be considered a behavior of a changeable magnetic field and 
velocity in the plasma of the inner Sun's atmosphere- from photosphere to lower corona. We assume that more appropriate and notable candidate of the topological structure that can be considered as a cause of quasi-periodical changes with various time intervals, among them the 3-4 CRP recurrence, is a diffusion of current sheet in the Sun's atmosphere.

Moreover, it is worth to note that the process which is responsible for changes of the magnetic field topology is a turbulent magnetic reconnection (e.g. [14]). For this process Parker [15] and Sweet [16] have proposed independently the first analytical model- when strong magnetic field lines diffuse through the plasma then reconnection can occur. Thus, it might be very useful to study the time-scales of the solar magnetic reconnection.

Our purpose in this paper is twofold: (1) to examine features of the 3-4 CRP recurrence of the 27-day variations of the GCR intensity, parameters of SA and SW using experimental data, (2) to study the topological structure of the Sun due to the temporal changes of the coronal green line intensity.

\section{Recent observational data}

To investigate the properties of the 3-4 CRP periodicity using the recent experimental data, we consider daily data in the period of 2009-2017 as the end of the extended solar minimum 2006-2009 (e.g. [17]) and a part of solar cycle 24. We analyze different parameters measured on the Earth and in the nearest space around the Earth's orbit, among them the GCR intensity measured by Rome neutron monitor, solar wind speed (SWs), solar wind plasma temperature (SWt), the strength (B) of the heliospheric magnetic field (HMF), sunspot number (SSN) and $10.7 \mathrm{~cm}$ solar radio flux (F10.7). To find amplitudes of the 27-day variations of different parameters we use harmonic analysis method (e.g. [18]). Figure 1 shows the sequence of seven 3-4 CRP, as an example, starting from February 2011, occurring in the all described data. One can see, that the 3-4 CRP recurrence is clearly visible in the amplitudes of the 27-day variations of the GCR intensity, SW speed, SW plasma temperature, the HMF strength, sunspot number and solar radio flux.

We consider the 3-4 CRP cycling as rather sporadic effect. It can be present during more than two years, but after it vanishes, and appears again after few rotations. We suppose that this quasi- recurrence is an universal feature of solar activity associated with the solar dynamo and differential rotation of the Sun - once in a while disrupted by other processes, not very easily visible, nevertheless, constantly remaining.

\section{Examine the solar corona and discussion}

Mursula and Vippola [19] studying SW changes measured by various space probes showed that 1.3-years periodicity, i.e. a recurrence found in [20], is weaker in high heliolatitudes and stronger in the equatorial region. They also reported, that 1.7-years periodicity, i.e. a recurrence found in [21], behaves opposite, being stronger in mid -latitudes. In [22] there is a statement, that the manifestation of the rotation of the innermost part of sub-photospheric zones might be reflected in the solar corona behavior. 

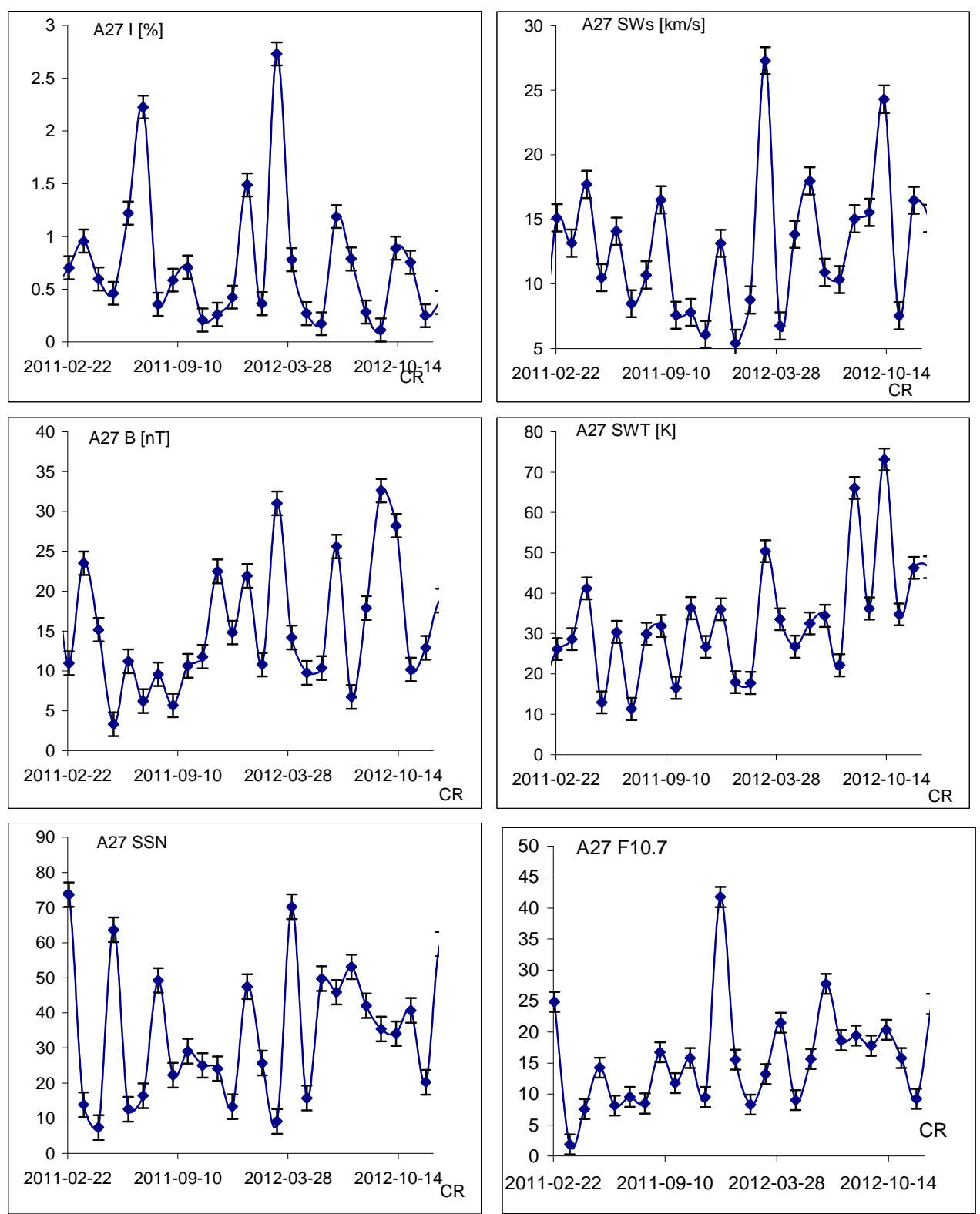

Fig. 1 Sequences of 3-4 CRP quasi-recurrence in the 2011-2012 of amplitudes of the 27-day variation of (a) the GCR intensity by Rome neutron monitor (I), A27 I, (b) the solar wind speed (SWs), (c) the interplanetary magnetic field strength (B), (d) the solar wind plasma temperature (SWT), (e) the sunspots number $(\mathrm{SSN})$, (f) $10.7 \mathrm{~cm}$ solar radio flux (F10.7). On the abscissa the unit is Carrington rotation $(\mathrm{CR})$

Thus, to track the structures hidden in the solar magnetic field we study changes of the coronal green line intensity (CGLI) with wavelength of $530.3 \mathrm{~nm}$, emitted by ionized iron Fe XIV (e.g. [23-24]) in the period 1958-2008. CGLI gives an insight in the time-latitudinal spreading of the coronal brightness.

We have calculated amplitudes of the 27-day variation of CGLI at all points over the solar disc, starting from the North pole in the eastern direction, with $5^{0}$ intervals. Figures $2 \mathrm{a}-\mathrm{h}$ show the sequence of the 3-4 CRP in the periods 1980-1982 and 1996-1998, occurring in CGLI at 
different points of solar disc (as it was found for the other data, as GCR, SW and SA parameters-compare with Fig. 2 and 3 in [12]).
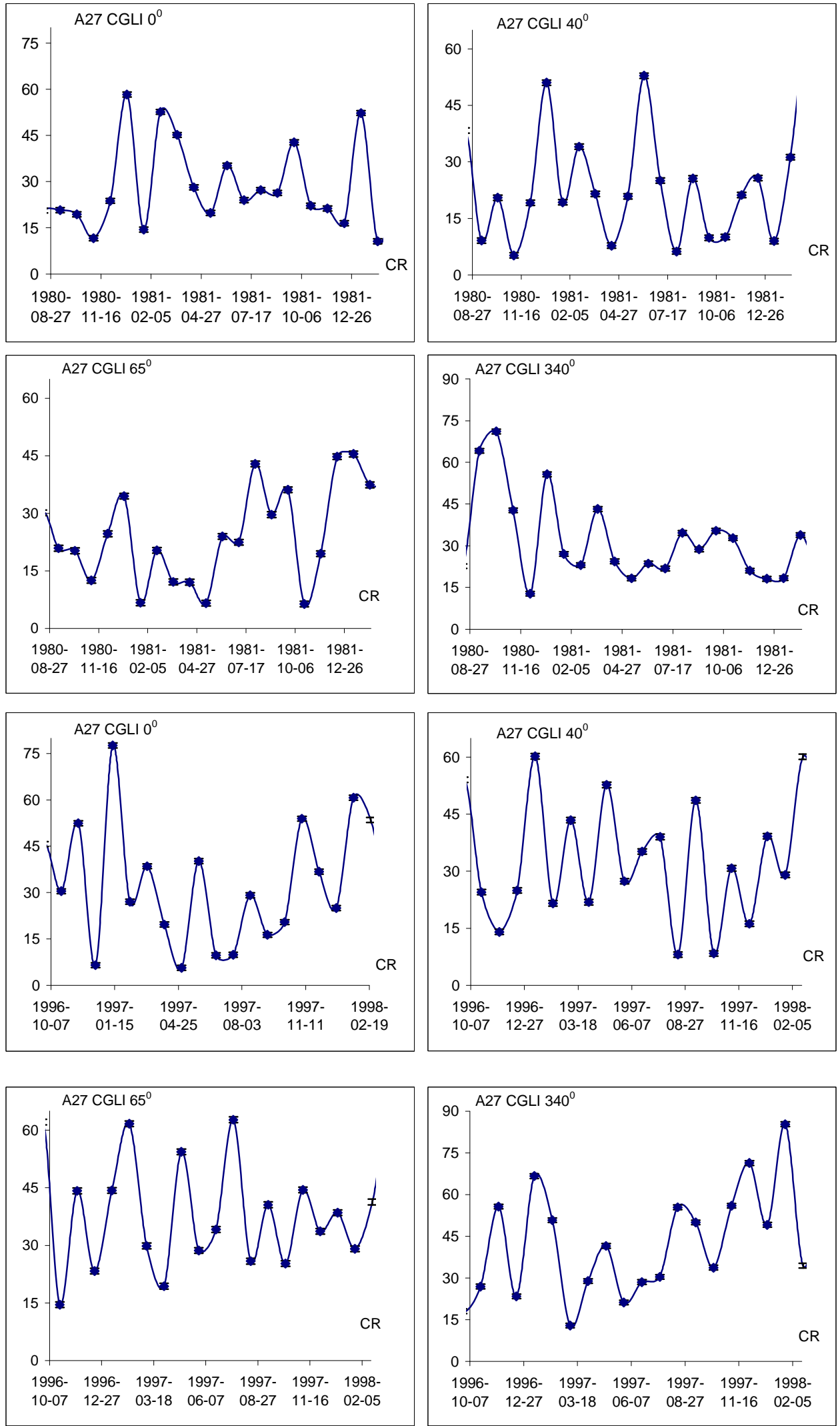
Fig. 2a-h Sequences of the 3 -4 CRP quasi-recurrence in 1980-1982 (a-d) and 1996-1998 (e-h) of the amplitudes of the 27- day variation of the coronal green line intensity for (a) $0^{0}$ (CGLI $0^{\circ}$ ), (b) $40^{\circ}$, (c) $65^{\circ}$, (d) $340^{\circ}$ in absolute coronal units (acu). On the abscissa the unit is Carrington rotation (CR).

We have found that the 3-4 CRP quasi-periodicity is mostly clearly visible in midlatitudes and near the polar regions, especially the North hemisphere; possibly this is an indication of the existence of stable North-South asymmetry of solar dynamo, while this recurrence does not exhibit in the equatorial regions.
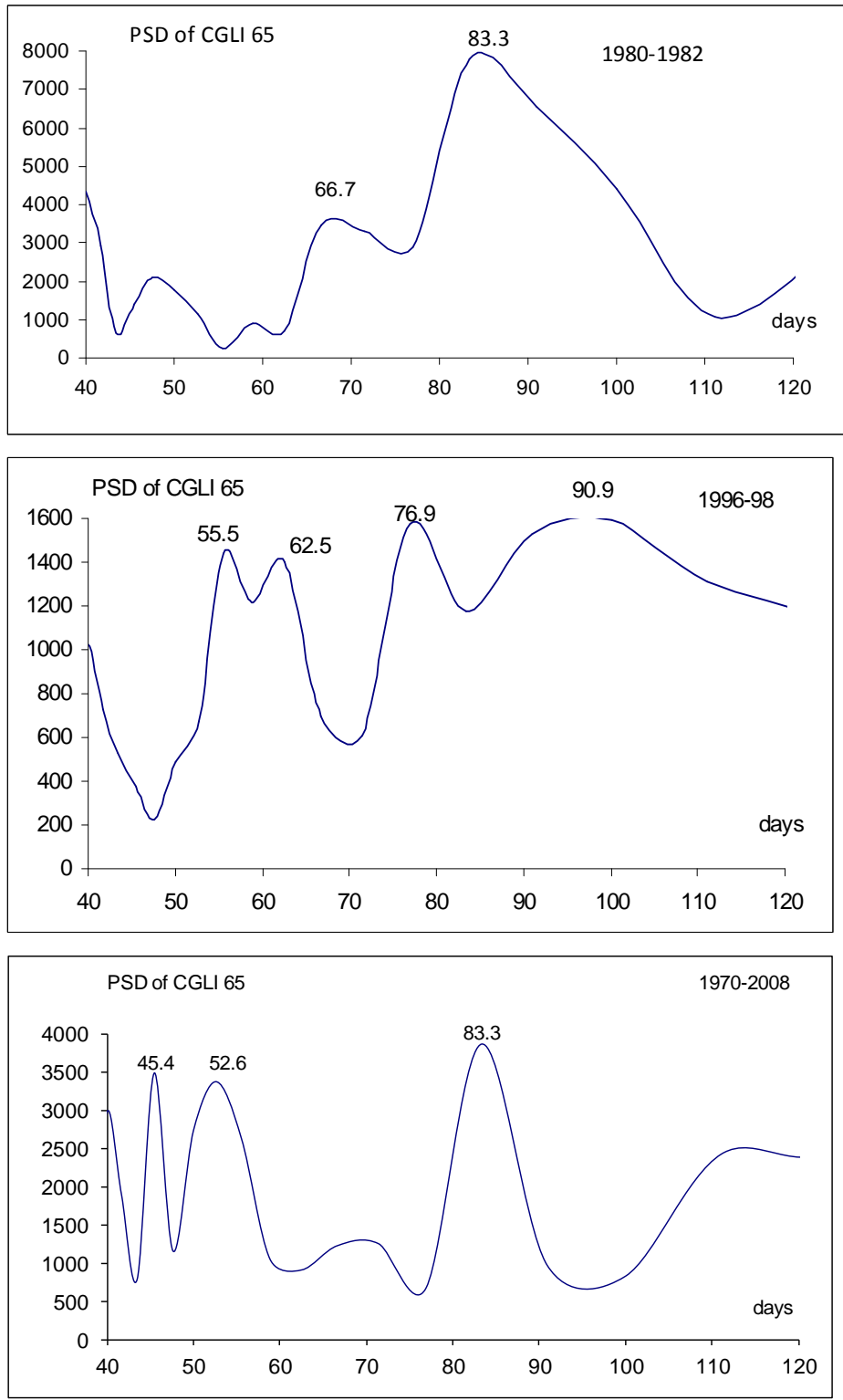

Fig. 3a-c PSD of daily data of CGLI for $65^{\circ}$ (as an example) for the time intervals: 1980-1982 (a), 1996-1998 (b) and 1970-2008 (c) with periodicities from 40 days to 120 days.

To confirm the above results we also calculated the power spectrum densities (PSD) using daily data of considered parameters. Figure 3 shows periodicities from 40 to 120 days revealed in CGLI in various points of solar disc in the periods 1980-1982, 1996-1998 and for comparison 
the longer period of 1970-2008. For each time interval the periodicity close to the duration of 3 Carrington rotations period is clearly visible.

It is worth to underline, that in [25] it was shown that almost all sunspots at the end of the Maunder minimum were located in the southern hemisphere and the Grand Minima occurrence and North-South asymmetry of solar activity might be related [7]. In [26] it was found that the distribution density of the background magnetic field also shows North-South asymmetry.

\section{Conclusions}

1. We confirm an existence of clearly established quasi-periodicity with duration of three to four Carrington rotations period (3-4 CRP) in the changes of the amplitudes of the 27-day variations of GCR intensity, parameters of solar wind and solar activity.

2. The 3-4 CRP recurrence is shaped by the combined: solar dynamo and differential rotation. Particularly, the most appropriate candidate of the topological structure which can be considered as a cause of the 3-4 CRP quasi-periodicity is diffusion of a current sheet in the Sun's lower atmosphere. It can be assumed that a study of an evolution of current sheet in lower atmosphere of the Sun can shed light on the nature of the spatially and temporally changeable topological structure responsible for the 3-4 CRP recurrence of the amplitudes of the 27-day variations of GCR intensity, and different parameters of solar activity and solar wind. We believe that there is a spectrum of the topological structure of the magnetic current sheet on the Sun's photosphere being responsible for different quasi- periodical changes, including the 3-4 CRP recurrence. Thus, STS should be very complex pattern containing a broad modes of oscillations responsible for various types of quasi-periodicities. Hence, it is possible to find an evidence that any alternation: periodical or non-periodical in GCR intensity and in parameters of solar activity and solar wind is strongly related with temporal changes of the various scales of STS.

4. We show that the 3-4 CRP quasi-periodicity in the amplitudes of the 27-day variation of the coronal green line intensity is clearly visible mostly in mid-latitudes and near the polar regions, especially in North hemisphere. We believe that it confirms an existence of the stable North-South asymmetry of solar dynamo.

\section{Acknowledgements}

Authors acknowledge: the Investigators of the Rome neutron monitor, http://webusers.fis.uniroma3.it/svirco/pag_2.html, and Researcher of: Neutron Monitor Database, http://www.nmdb.eu/; NASA/Goddard Space Flight Center, http://omniweb.gsfc.nasa.gov/; The National Geophysical Data Center, ftp://ftp.ngdc.noaa.gov/STP/SOLAR\_DATA/. This work was supported by the Polish National Science Centre, decision number DEC-2016/22/E/HS5/00406.

\section{References}

[1] Ruzmaikin, A.A. (1985), The solar dynamo, Solar Physics, 100, 125-140.

[2] McCracken, K.G., J. Beer, F. Steinhilber, and J. Abreu (2013), A Phenomenological Study of the Cosmic Ray Variations over the Past 9400 Years, and Their Implications Regarding Solar Activity and the Solar Dynamo, Solar Physics, 286, 609-627.

[3] Simoniello, R., K. Jain, S.C. Tripathy, S. Turck-Chieze, C. Baldner, W. Finsterle, F. Hill, and M. Roth (2013), The Quasi-Biennial Periodicity As a Window on the Solar Magnetic Dynamo Configuration, Astrophysical Journal, 765:100. 
[4] Bazilevskaya, G., A.-M. Broomhall, Y. Elsworth, and V.M. Nakariakov (2014), A Combined Analysis of the Observational Aspects of the Quasi-biennial Oscillation in Solar Magnetic Activity, Space Sci. Rev., doi 10.1007/s11214-014-0068-0.

[5] Howe, R., J. Christensen-Dalsgaard, F. Hill, R.W. Komm, R.M. Larsen, J. Schou, M.J. Thompson, and J. Toomre (2000), Dynamic Variations at the Base of the Solar Convection Zone, Science, 287, 2456-2460.

[6] Howe, R., R. Komm, F. Hill, J. Christensen-Dalsgaard, T.P. Larson, J. Schou, M.J. Thompson, and J. Toomre (2011), Rotation-rate variations at the tachocline: an update, Journal of Physics: Conference Series, 271012075

[7] Olemskoy, S.V., A.R. Choudhuri, and L. L. Kitchatino (2013), Fluctuations in the Alpha-Effect and Grand Solar Minima, arXiv:1305.2660

[8] Ruzmaikin A.A., A.C. Cadavid, and J. Lawrence (2008), Quasi-periodic patterns coupling the Sun, solar wind and the Earth, J. Atmos. Sol.-Terr. Phys., 70, 212-217.

[9] Kudela, K. (2013), Space weather near Earth and energetic particles: selected results, Journal of Physics: Conference Series, 409, 012017.

[10] Abreu, J.A., J. Beer, A. Ferriz-Mas, K.G. McCracken, and F. Steinhilber (2012), Is there a planetary influence on solar activity?, Astron. Astrophys., 548, A88.

[11] Gil, A., and M.V. Alania (2011), Cycling The rigidity spectrum of the harmonics of the 27-day variation of the galactic cosmic ray intensity in different epochs of solar activity: 1965-2002, J. Atmos. Sol.-Terr. Phys., 73, 294-299.

[12] Gil, A., and M.V. Alania (2012), Cycling changes in the amplitudes of the 27-day variation of the galactic cosmic rays intensity, Solar Physics, 278, 447-455.

[13] Gil, A., and M.V. Alania (2013), $\alpha$ - $\omega$ effect and peculiarities of the 27-day variations of the galactic cosmic rays intensity, solar wind and solar activity parameters, Adv. Sp. Res., 52, 951-956.

[14] Browning, Ph., and A. Lazarian (2013), Notes on Magnetohydrodynamics of Magnetic Reconnection in Turbulent Media, Space Sci. Rev., 178, 325-355.

[15] Parker, E.N. (1957), Sweet's mechanism for merging magnetic fields in conducting fluids, J. Geophys. Res. 62, 509-520.

[16] Sweet, P.A. (1958), The neutral point theory of solar flares, in Electromagnetic Phenomena in Cosmical Physics, ed. by B. Lehnert., Conf. Proc. IAU Symposium, 6, 123-134.

[17] McCracken, K.G., and J. Beer (2014), Comparison of the extended solar minimum of 2006-2009 with the Spoerer, Maunder, and Dalton Grand Minima in solar activity in the past, J. Geophys. Res. Space Physics, 119, 2379-2387.

[18] Kincaid, D.R., and E.W Cheney (2009), Numerical analysis: mathematics of scientific computing, third ed., AMS Bookstor.

[19] Mursula, K., and J. H. Vilppola (2004), Fluctuations of the Solar Dynamo Observed in the Solar Wind and Interplanetary Magnetic Field at 1 AU and in the Outer Heliosphere, Solar Physics, 221, 337-349.

[20] Richardson, J., K. Paularena, J.W. Belcher, and A. Lazarus (1994), Solar wind oscillations with a 1.3 year period, Geophys. Res. Lett., 21, 1559-1560.

[21] McIntosh, P., R. Thompson, and E. Willock (1992), A 600-day periodicity in solar coronal holes, Nature, 360, 322-324.

[22] Badalyan, O.G. (2010), Two types of differential rotation of the solar corona, New Astronomy, 15, 135-143.

[23] Rybak, J. (1994), Rotational characteristics of the green solar corona: 1964-1989, Solar Physics, 152, 161-166

[24] Dorotovic, I., M. Minarovjech, M. Lorenc, and M. Rybanský (2014), Modified Homogeneous Data Set of Coronal Intensities, Solar Phys., 289, 2697-2703

[25] Ribes, J. C., and E. Nesme-Ribes (1993), The solar sunspot cycle in the Maunder minimum AD1645 to $A D 1715, A \& A, \mathbf{2 7 6}, 549-563$.

[26] Obridko, V.N., V.E. Certoprud, and K.M. Kuzanyan (2014), North-South Asymmetry in the Distribution of Solar Background Magnetic Field, Solar Physics, 289, 2867-2878. 